\title{
The $n$-queens problem in higher dimensions
}

\author{
Jeremiah Barr and Shrisha Rao \\ Shrisha Rao received his Ph.D. in Computer Science from the University of Iowa. He \\ is a native of India and spent a large part of his life in the United States. His research \\ interests include safety in distributed systems, centrality in tree graphs, and the social \\ consequences of information technology. \\ Jeremiah Barr is an undergraduate at Mount Mercy College in Cedar Rapids, Iowa. \\ He is studying Computer Science and Mathematics. He is also a part-time business \\ software developer. His fields of interest include Artificial Intelligence.
}

\section{Introduction}

The 8-queens problem is a well-known chessboard problem, whose constraints are to place eight queens on a normal chessboard in such a way that no two attack each other, under the rule that a chess queen can attack other pieces in the same column, row, or diagonal. This problem can be generalized to place $n$ queens on an $n$ by $n$ chessboard, otherwise known as the $n$-queens problem. The mathematicians Gauss and Polya studied this problem [3], and Ahrens [1] showed that for all $n \geq 4$, solutions exist. This problem can be further generalized to $d$ dimensions, where two queens attack one another if they lie on a common hyperplane. It can then be described as the $n$-queens problem in $d$ dimensions. (The traditional 8-queens problem, as described above, is 2-dimensional.)

The $n$-queens problem is classically considered a theoretical one, but has also been studied [5] for its many applications: in distributed memory storage schemes [4], VLSI test-

In einem bekannten Schachproblem wird die Frage gestellt, wie man acht Damen so auf einem Schachbrett aufstellen kann, dass sie sich gegenseitig nicht schlagen können. Dieses Problem wurde schon von Gauss untersucht. Es kann auf ein $n \times n$-Schachbrett verallgemeinert werden, wobei $n \geq 4$ gilt. Im vorliegenden Beitrag wird in einem $n$-reihigen $d$-dimensionalen Schachraum das folgende Problem untersucht: Wieviele Damen müssen mindestens aufgestellt werden, um alle Brettpositionen anzugreifen? Für $d \geq 3$ reichen $n$ Damen im Allgemeinen nicht aus. Eine untere Schranke für die Mindestanzahl von Damen wird angegeben. Ausserdem wird gezeigt, dass es für jede natürliche Zahl $k$ höherdimensionale Schachräume gibt, in denen $n^{k}$ Damen nicht ausreichen, um alle Felder anzugreifen. 
ing [6], deadlock prevention in computer systems [8], and others. As a canonical problem in constraint satisfaction, the problem is also approached using neural networks [7], and also studied as a standard candidate for the backtracking (depth-first search) method.

It is trivial that for all $n \geq 4, d \geq 3$, there always is a way of placing $n$ queens in a $d$ dimensional board of size $n$ so that no two attack each other. This follows from the result [1] that for all $n \geq 4, n$ queens can be placed on a regular 2-dimensional board.

We previously performed a computational analysis [2] of the $n$-queens problem in higher dimensions, by counting the number of ways in which $n$ queens can be placed. This analysis seemed to indicate that there were non-attacking queens solutions in $d$ dimensions that could not be projected onto subspaces, and led us to investigate the following question:

LEAST What is the least number of queens that would be necessary to attack every position in a $d$-dimensional board of size $n$ ?

Certain simple cases are easy to analyse. For instance, if $n=3$, the least number of queens that can attack all board positions on a $d$-dimensional board is 1 for all $d \geq 1$. Similarly, when $d$ is 2 , i.e., on the regular board of size $n$, we obviously need no more than $n$ queens, and in fact, fewer suffice. Our present work gives the first general lower bound on the number of queens needed, for all $n$ and $d$.

\section{The queens problem}

\subsection{In two dimensions}

On a 2-dimensional grid, a queen can attack along the two axes $X$ and $Y$, and along two diagonals. For a queen located at Cartesian coordinates $\left\langle q_{1}, q_{2}\right\rangle$, the axes are given if $x=$ $q_{1}$ and $y=q_{2}$. The diagonals are $x-q_{1}=y-q_{2}$ and $x-q_{1}=q_{2}-y$, and these are the 1 - and 2-dimensional attack lines, respectively.

\subsection{In $d$ dimensions}

Consider a $d$-dimensional hypercube. The vertices of such a hypercube can be addressed by bit strings of length $d$ - the $2^{d}$ vertices of the hypercube can be addressed by $000 \ldots 0$ through $111 \ldots 1$.

Two vertices in a $d$-dimensional hypercube are adjacent if their addresses differ in just one bit position. If they differ in $k \leq d$ positions, they lie on a $k$-dimensional diagonal. Therefore, the longest-length diagonal connects a vertex with its polar reciprocal (the opposite vertex, whose address bits are all inverses of the corresponding bits).

A chess board of size $n$ is similar to a hypercube, except that instead of just two values, each position in the string takes $n$ values, from 0 through $n-1$.

A queen in a $d$-dimensional chess space therefore has a position given by a $d$-dimensional vector $\left\langle q_{1}, q_{2}, \ldots, q_{d}\right\rangle$, where $0 \leq q_{k} \leq n-1$.

The equations for the $d$-dimensional attack lines for a queen at location $\left\langle q_{1}, q_{2}, \ldots, q_{d}\right\rangle$ in a $d$-dimensional chess hyperspace are of the form

$$
\pm\left(x_{1}-q_{1}\right)= \pm\left(x_{2}-q_{2}\right)=\ldots= \pm\left(x_{d}-q_{d}\right) .
$$


There are obviously $2^{d}$ such equations in all. However, noting that changing the signs on all the terms gives us a new equation with the same meaning as the one changed, there are $2^{d-1}$ equations that are distinct.

Lemma 2.1. A queen in $d$-space can also be considered as having attack vectors of the form $\left\langle\delta_{1}, \delta_{2}, \ldots, \delta_{d}\right\rangle, \delta_{k} \in\{-1,0,1\}$, with the constraint that $\left\{\delta_{1}, \delta_{2}, \ldots, \delta_{d}\right\} \neq\{0\}$.

Proof. A component $\delta_{k}$ of an attack vector represents a queen's movement in dimension $k$ along one attack line. Such movement can be in two directions ("forward" or "backward", so to speak) which we can represent as +1 or -1 ; if there is no movement in dimension $k$, then $\delta_{k}=0$.

It is not possible for all components to be zero, i.e., $\left\{\delta_{1}, \delta_{2}, \ldots, \delta_{d}\right\} \neq\{0\}$, because an attack vector must have the queen moving along at least one dimension - a zero vector denotes complete lack of movement from the queen's current position.

Each attack line for a queen is composed of the maximum range of the queen's movement along two attack vectors (one in each direction from the queen's location) such that their sum results in the zero vector $\left\langle 0_{1}, 0_{2}, \ldots, 0_{d}\right\rangle$. Hence, the number of attack lines is half the number of attack vectors.

Remark 2.2. The board positions at a scalar distance $s>0$ along a queen's attack vector are given by:

$$
s\left\langle\delta_{1}, \delta_{2}, \ldots, \delta_{d}\right\rangle+\left\langle q_{1}, q_{2}, \ldots, q_{d}\right\rangle .
$$

Theorem 2.3. A queen in d-space has a total of

$$
\frac{3^{d}-1}{2}
$$

attack lines.

Proof. We know from Lemma 2.1 that each coordinate of a queen's attack vector can have one of 3 values. An attack vector also has $d$ components (as it is a vector in $d$-space). However, the zero vector where the queen does not move in any direction is ruled out, as pointed out in the lemma.

Therefore, the queen has $3^{d}-1$ attack vectors. Since the number of attack lines is half the number of attack vectors, the number of attack lines is $\frac{3^{d}-1}{2}$.

\subsection{LEAST}

For the question LEAST stated in Section 1, we first consider the following

Lemma 2.4. A queen in any $d$-dimensional chess space of size $n$ can attack at most $n$ board positions (including the one it holds) along any one attack line.

Proof. By Remark 2.2, we can find a board position attacked by the queen along an attack vector by taking the sum of queen's position and the product of the attack vector as given in Lemma 2.1 and a scalar distance $s>0$ from the queen's position. 
The individual $k$-dimensional coordinate of a board position attacked by a queen is given by $s \delta_{k}+q_{k}$. We know that the chess space itself does not have any position with a coordinate greater than $n-1$ or less than 0 . Therefore, any attack vector terminates when $s \delta_{k}+q_{k}=0$ or $s \delta_{k}+q_{k}=n-1$, for any coordinate $k$. Therefore, $s$ can take at most $n$ values since at least one of the $\delta_{k}$ must be non-zero and it can only have $n$ values along that coordinate.

From this and Theorem 2.3, we get the following

Lemma 2.5. A queen in a d-dimensional chess space of size $n$ can attack no more than $\frac{n\left(3^{d}-1\right)}{2}$ board positions.

Note also that there are $n^{d}$ board positions in a $d$-dimensional chess space of size $n$. Therefore, by dividing the number of board positions by the maximum number of positions attacked by a queen, we have the following

Theorem 2.6. The least number of queens necessary to attack all positions in a d-dimensional chess space of size $n$ is no less than

$$
\frac{2 n^{d-1}}{3^{d}-1}
$$

In deriving this result, we have assumed that every queen is able to attack as many board positions as possible along every attack line, and that no two queens attack the same board position. This is obviously an overestimate, so the above expression is a lower bound subject to refinement using more intricate analyses.

No more than $n$ non-attacking queens can be placed on a two-dimensional board (since there are only $n$ rows or columns, and every queen must be on a separate row and column). However, Theorem 2.6 shows us that this is not true in higher-dimensional spaces. Specifically, we have the following

Corollary 2.7. When $d \geq 3$, it is not always possible to attack all board positions on a board of size $n>3$ using $n$ queens.

Proof. Given Theorem 2.6, we know that for any values $n$ and $d$ such that

$$
\frac{2 n^{d-1}}{3^{d}-1}>n,
$$

which gives, upon simplification,

$$
2 n^{d-2}>3^{d}-1
$$

it is impossible to attack all board positions using just $n$ queens.

Similarly, we can show that it is always possible to find an $n$ large enough that given a certain $d$, it is not possible to attack all board positions using $n^{k}$ queens, where $k<d-1$.

Corollary 2.8. If $2 n^{d-k-1}>3^{d}-1$, it is not possible to attack all board positions using $n^{k}$ queens. 
It is therefore also possible to always find a chess space of large enough dimension and size so that for any $k, n^{k}$ queens do not suffice; alternatively, for any $d>2, n^{d-2}$ queens do not suffice for all but a finite number of $n$.

\section{Suggestions for further work}

Based on the work presented here, the authors see a likely future result being the derivation of an exact expression for the number of queens necessary to attack all positions in a $d$ dimensional board of size $n$. This may prove to be difficult, however, so an easier effort should be one directed towards a better lower bound than Theorem 2.6 provides. Likewise, a non-trivial upper bound should also be obtainable.

Related to these, of course, are the more standard problems relating to the enumeration of the possible solutions in case of a $d$-dimensional board of size $n$. Algorithms for placement of queens in higher-dimensional spaces (a trivial problem when there are no more queens than indicated by Theorem 2.6) are also worth investigating. Considering the slew of applications of the standard 2-dimensional problem, it also remains to be seen what applications can be made of the higher-dimensional analogue and results therein.

\section{Acknowledgement}

The authors would like to thank K.R. Knopp for useful discussions on this topic.

\section{References}

[1] Ahrens, W.: Mathematische Unterhaltungen und Spiele. Berlin 1910.

[2] Barr, J.; Rao, S.: Some Observations about the $n$-Queens Problem in Higher Dimensions. Midwest Instruction and Computing Symposium, Morris, Minnesota, 2004. Available on the web at http://www.micsymposium.org/mics_2004/Barr.pdf.

[3] Polya, G.: Über die “doppelt-periodischen” Lösungen des $n$-Damen-Problems. In: Ahrens, W.: Mathematische Unterhaltungen und Spiele II, 364-374, 1918.

[4] Erbas, C.; Tanik, M.M.: Storage Schemes for Parallel Memory Systems and the N-Queens Problem. The 15th ASME ETCE Conference, Computer Applications Symposium, Houston, Texas, January 26-30, 1992.

[5] Erbas, C.; Sarkeshik, S.; Tanik, M.M.: Different Perspectives On the N-Queens Problem. CSC '92: Proceedings of the 1992 ACM Annual Conference on Communications, 99-108.

[6] Funabiki, N.; Kurokawa, T.; Ohta, M.: Binary Neural Networks For N-Queens Problems and Their VLSI Implementations. Control Cybernet. 31(2) (2002), 271-296.

[7] Funabiki, N.; Takenaka, Y.; Nishikawa, S.: A Maximum Neural Network Approach For N-Queens Problems. Biological Cybernetics 76 (1997), 251-255.

[8] Tanik, M.M.: A graph model for deadlock prevention, Ph.D. thesis, Texas A \& M University, 1978, UMI Order Number AAI7909241.

Jeremiah Barr

Dept of Computer Science

Mount Mercy College

Cedar Rapids, IA 52402, USA

e-mail: jrb62307@att.net

\author{
Shrisha Rao (Corresponding author) \\ IIIT-Bangalore \\ 26/C Electronics City \\ Bangalore 560 100, India \\ e-mail: srao@iitb.ac.in
}

